\title{
Neuropsychiatric morbidity in focal epilepsy
}

Sophia J. Adams, Terence J. O'Brien, John Lloyd, Christine J. Kilpatrick, Michael R. Salzberg and Dennis Velakoulis

\section{Background}

Previous work has identified elevated prevalence rates for psychiatric disorders in individuals with medically refractory focal epilepsy, particularly temporal lobe epilepsy. Many studies were undertaken before the advent of video electroencephalogram monitoring (VEM) and magnetic resonance imaging (MRI).

\section{Aims \\ To investigate which characteristics of the focal epilepsy syndromes are associated with the presence of depression} or psychosis.

\section{Method}

Three hundred and nineteen individuals with focal epilepsy admitted for VEM were seen over an 11-year period. The lifetime history of depression and psychosis, epileptic site, laterality and type of lesion were determined by clinical assessment, VEM and MRI scan.

\section{Results}

There was a significant association between the prevalence of depressive symptoms and non-lesional focal epilepsy. There were no significant differences in prevalence of neuropsychiatric disorders between the groups with temporal lobe epilepsy and those with extratemporal lobe epilepsy.

\section{Conclusions}

These findings contrast with previous findings in smaller cohorts. The association between non-lesional focal epilepsy and depression may be due to the effects of a more diffuse epileptogenic area.

\section{Declaration of interest}

S.J.A. was supported for 1 year by a UCB Pharma Neurosciences scholarship awarded by the Epilepsy Society of Australia in 2004.
The seminal works of Gibbs, ${ }^{1}$ Slater \& Beard ${ }^{2}$ and Flor-Henry ${ }^{3}$ on the schizophrenia-like psychoses of epilepsy have formed the foundation for the modern view that temporal lobe epilepsy and psychiatric illness are closely related. This relationship was investigated predominantly prior to the advent of video electroencephalogram monitoring (VEM) and magnetic resonance imaging (MRI) for the characterisation of seizure focus, seizure type and identification of the underlying lesion. For example, 66\% of the studies identified in five major reviews of schizophrenia-like psychoses of epilepsy, ${ }^{4-8}$ were published prior to 1986 .

There is a lack of consistency in findings across studies reflecting methodological differences and changing diagnostic classifications within neurology and psychiatry. Studies differ in methods used for disease classification and case ascertainment. The majority of studies have been cross-sectional and retrospective. Sample sizes are generally less than 100 individuals. Only a few studies have accurate psychiatric diagnoses based on semistructured patient interview., ${ }^{9,10}$ Many describe 'psychopathology' in general ${ }^{11}$ or use rating scales. The subsequent heterogeneity of diagnostic categories hampers comparison.

It is generally accepted that individuals with focal epilepsy exhibit higher rates of psychopathology compared with those with generalised epilepsy, neurological control groups, people with chronic non-neurological disorders and the general population. ${ }^{6,12}$ Reported prevalence rates for mental illness in those with medically refractory focal epilepsy are $44-88 \%{ }^{4}$ Depression and psychosis constitute the two most investigated and clinically relevant psychiatric disorders in people with focal epilepsy. There is also more recent evidence that the relationships between depression, psychosis and seizures may be more complex than previously imagined. Depression has been associated with the development of later seizures. ${ }^{13} \mathrm{~A}$ family history of epilepsy has been associated with the development of both epilepsy and psychotic disorders. ${ }^{14}$

Owing to the lack of recent research, our view of the relationship between psychopathology and epilepsy is dependent on increasingly less comparable literature. This may in part account for the ongoing lack of consensus regarding specific associations between temporal lobe epilepsy and psychiatric disorders, ${ }^{15-17}$ psychosis ${ }^{18}$ or depression. ${ }^{19-21}$ Issues which remain unresolved include whether individuals with temporal lobe epilepsy are at greater risk of developing psychiatric disorders than those with other forms of focal epilepsy, ${ }^{11}$ and whether the focal laterality or the presence of a defined lesion influence psychopathology. It has been suggested that the issue will not be resolved until data from large series of well-categorised individuals can be collected and studied. ${ }^{7}$ The current study seeks to redress this historical dissociation by investigating a large series of participants with focal epilepsy who have well-defined epilepsy semiology using VEM, MRI and detailed assessment of the psychiatric history.

\section{Method}

\section{Inclusion criteria}

The study group consisted of all people seen consecutively in the VEM unit at the Royal Melbourne Hospital between 1993 and 2004 who had a diagnosis of focal epilepsy and a clinical neuropsychiatric assessment. Individuals are admitted to the VEM primarily because of ongoing seizures despite anti-epileptic drug therapy. The purpose of the admission is to obtain a more comprehensive diagnostic understanding of their condition, including neuropsychiatric aspects, and to better define suitable treatment options that may involve the consideration of whether surgical intervention is appropriate.

All individuals $(n=482)$ admitted over the study period with a discharge diagnosis of focal epilepsy were reviewed by examination of their medical record, discharge letters and discussion with their treating epileptologists. Ethical approval for this retrospective audit of patient files was obtained from the human research and ethics committee of the Royal Melbourne Hospital. Fifty-four individuals were excluded because of insufficient evidence for focal epilepsy. Of the 428 individuals confirmed to 
have focal epilepsy, 319 had undergone a clinical neuropsychiatric assessment at the time of their epilepsy assessment. Information regarding individuals' demographic data, neuropsychiatric diagnoses, epilepsy classification, seizure laterality and MRI findings were collated.

\section{Neurological assessment and classification of focal epilepsy type}

All in-patient comprehensive epilepsy evaluations consisted of a thorough clinical history, continuous VEM over 1-3 weeks for seizure location and 1.5 T MRI epilepsy protocol brain scans, which included whole brain coronally acquired volumetric seizures. ${ }^{22}$ Where clinically indicated, single photon emission tomograhy (SPECT) and fluorodexyglucose positron emission tomography (FDG-PET) studies were also performed to clarify the epileptic focus. There were no changes in MRI procedures and in the scanner used over the time of this study. A consensus neurological diagnosis, informed by the International League Against Epilepsy classification system, ${ }^{23}$ was determined at a weekly epilepsy clinical review meeting attended by three epileptologists, the neuropsychiatrist (J.L.), a neuropsychologist, epilepsy fellows, electroencephalogram (EEG) technicians and a neuroradiologist.

The focal epilepsies were divided into temporal lobe epilepsy and extratemporal lobe epilepsy. The group with temporal lobe epilepsy were further classified into the following three diagnostic subgroups: mesial temporal lobe epilepsy, who had mesial temporal sclerosis on MRI and an ipsilateral focus on VEM; non-lesional temporal lobe epilepsy, who had no imaging evidence of pathology but a clear temporal localisation on VEM; and lesional temporal lobe epilepsy when there was an epileptogenic lesion in the temporal lobe on MRI (other than mesial temporal sclerosis, i.e. 'foreign tissue lesion') and an ipsilateral focus on VEM. The extratemporal lobe epilepsy group was divided into those with and without an epileptogenic lesion on MRI. The laterality of the epilepsy was determined by agreement between the inter-ictal, ictal EEG findings and any pathology seen on MRI and where available PET and/or SPECT.

Seven individuals with extratemporal lobe epilepsy were excluded because of missing MRI data. This left 312 participants in the study. Laterality could not be ascertained in eight people on the VEM and these were therefore excluded from the analysis of laterality.

\section{Neuropsychiatric assessments}

All people with focal epilepsy admitted for VEM were routinely referred for neuropsychiatry assessments. However, because of time limitations and neuropsychiatrist leave not all were assessed. The group assessed were more likely to have temporal lobe epilepsy $\left(\chi^{2}=7.7, P<0.05\right)$ than any other diagnosis but did not differ significantly from those not assessed by age, gender, laterality of focal site or whether they later had neurosurgery.

The aims of the assessment were to identify clinically relevant psychiatric morbidity which may impact upon treatment or confound the neurological presentation. They were undertaken at the time of neurological assessment by a senior neuropsychiatrist who interviewed the participants and sought corroborative history where appropriate. A minority were performed by other neuropsychiatrists directly under his supervision, including D.V. and S.J.A. The neuropsychiatric assessments, including a neuropsychiatric formulation and diagnoses informed by DSM-IV diagnostic criteria, ${ }^{24}$ were thorough and documented in detail at the time of assessment. The assessment was completed and documented prior to the clinical review meeting in which the epilepsy diagnosis was made.

All neuropsychiatric assessments were collated after the study period and reviewed by a second neuropsychiatrist using a standardised pro forma to record information. The review of these assessments focused on the identification of past or current history of depression and past or current history of psychosis, including post-ictal psychoses and interictal psychoses but excluding ictal psychoses which were temporally related to the occurrence of the seizure. Other minor psychiatric phenomena occurring peri-ictally were not included.

Eleven of the neuropsychiatric assessments were reviewed by a third clinician using the same criteria, with $88 \%$ concordance in these diagnoses. Ten neuropsychiatric assessments were re-examined 12 months after the original file review by the second neuropsychiatrist with a diagnostic concordance of $100 \%$.

\section{Statistical analyses}

We undertook the following analyses to ascertain the relationship between psychiatric diagnoses and epilepsy groups:

(a) prevalence of psychiatric disorder (depressive, psychotic symptoms and other) within the total group and within the epilepsy subgroups;

(b) tests of association (chi-squared test) between the psychiatric diagnosis and the epilepsy subgroups; these analyses compared psychiatric diagnosis for:

(i) temporal lobe epilepsy $v$. extratemporal lobe epilepsy

(ii) non-lesional focal epilepsy (temporal and extratemporal lobe epilepsy both without lesions) $v$. lesional focal epilepsy (mesial temporal sclerosis and both lesional temporal and extratemporal lobe epilepsy)

(iii) non-lesional focal epilepsy (temporal and extratemporal lobe epilepsy without lesions) $v$. mesial temporal sclerosis focal epilepsy and other lesional focal epilepsy (both temporal and extratemporal)

(c) a logistic regression analysis using the epilepsy subgroups as independent variables to predict the odds of having the diagnosis was undertaken for the groups:

(i) any psychiatric diagnosis

(ii) depression

(iii) psychosis.

\section{Results}

\section{General demographic data and epilepsy syndrome diagnoses}

Of the 312 participants who met inclusion criteria, 121 (39\%) were diagnosed with mesial temporal lobe epilepsy; 74 (24\%) with non-lesional temporal lobe epilepsy; 58 (19\%) with lesional temporal lobe epilepsy; 42 (13\%) with extratemporal lobe epilepsy with lesions; and 17 (5\%) with extratemporal lobe epilepsy without lesions. The lateralisation of the seizure focus was left-sided in $48 \%$, right-sided in $42 \%$ and bilateral independent foci were present in $7 \%$. The seizure focus could not be lateralised in 8 participants $(3 \%)$. There were no significant differences between groups in age, gender, marital, employment status or laterality of foci.

The basic demographic and clinical details for each of the epilepsy syndromes is given in Table 1. Non-mesial temporal sclerosis lesions were detected on the MRI in 100 (32.1\%) participants where lesional epilepsy could be determined. The nature 
Table 1 Descriptive demographics of epilepsy groups

\begin{tabular}{|c|c|c|c|c|c|c|}
\hline & \multirow[b]{2}{*}{$\begin{array}{l}\text { All focal epilepsy } \\
\qquad(n=319)\end{array}$} & \multicolumn{3}{|c|}{ TLE } & \multicolumn{2}{|c|}{$\mathrm{ETLE}^{\mathrm{a}}$} \\
\hline & & $\begin{array}{c}\text { MTS } \\
(n=121)\end{array}$ & $\begin{array}{l}\text { Other lesions } \\
\qquad(n=58)\end{array}$ & $\begin{array}{l}\text { Non- lesional } \\
\quad(n=74)\end{array}$ & $\begin{array}{l}\text { Lesional } \\
(n=42)\end{array}$ & $\begin{array}{c}\text { Non-lesional } \\
\quad(n=17)\end{array}$ \\
\hline Average age, years & 36.5 & 37.0 & 35.5 & 36.8 & 37.7 & 34.5 \\
\hline Male, $n(\%)$ & $133(41.7)$ & $50(41.3)$ & $21(36.2)$ & $32(43.2)$ & $18(42.9)$ & $9(52.9)$ \\
\hline Never married, $n$ (\%) & $149(46.7)$ & $51(42.1)$ & $28(48.3)$ & $35(47.3)$ & $19(45.2)$ & $10(58.8)$ \\
\hline Unemployed, n (\%) & $120(37.6)$ & $47(38.8)$ & $22(37.9)$ & $21(28.4)$ & $18(42.9)$ & $6(35.3)$ \\
\hline Right/left/Bilateral, $n$ & $135 / 154 / 22$ & $51 / 65 / 4$ & $27 / 25 / 6$ & $27 / 38 / 4$ & $21 / 18 / 3$ & $4 / 8 / 4$ \\
\hline Ever given a psychiatric diagnosis, $n(\%)$ & $185(58)$ & $61(50.4)$ & $30(51.7)$ & $53(71.6)$ & $26(61.9)$ & $10(58.8)$ \\
\hline Ever depressed, $n$ (\%) & $104(32.6)$ & $32(26.4)$ & $16(27.6)$ & $31(41.9)$ & $15(35.7)$ & $7(41.2)$ \\
\hline Ever psychotic, $n(\%)$ & $22(6.9)$ & $10(8.3)$ & $5(8.6)$ & $4(5.4)$ & $2(8.6)$ & $1(4.8)$ \\
\hline
\end{tabular}

of these lesions were tumours, benign, malignant and dysembryoplastic neuroepithelial tumours $(n=23)$, encephalomalacia $(n=26)$, cavernomas $(n=10)$, dysplasias and other developmental abnormalities $(n=29)$ and others $(n=12)$ including dermoid and epidermoid cysts and arteriovenous malformations.

\section{Prevalence of any psychiatric disorder}

Fifty-eight per cent of participants were diagnosed as having a current or past history of a psychiatric disorder, with some individuals meeting the criteria for more than one disorder. The nature of the psychiatric disorder was depression in $32.6 \%$, psychosis in $7.2 \%$ and other psychiatric disorders in $36.1 \%$. The other psychiatric disorders included anxiety in $6.9 \%$, substance misuse or dependence in $3.1 \%$, somatoform disorders in $4.7 \%$, personality disorders in $13.8 \%$, more than one psychiatric diagnosis in $4.7 \%$ and other disorders in $2.8 \%$. Individuals in this 'other psychiatric disorders' group were evenly spread between the epilepsy subgroups. Rates for depression, psychosis and other psychiatric diagnoses did not differ significantly between males and females.

There were no significant differences in the prevalence of any psychiatric disorder between groups with temporal lobe epilepsy (57\%) v. extratemporal lobe epilepsy $(62.1 \%)\left(\chi^{2}=0.58, P=0.49\right)$. People with non-lesional epilepsy (both temporal and extratemporal lobe epilepsy groups) had a significantly higher prevalence of psychiatric disorders (69.2\%) than those with lesional epilepsy (mesial, temporal and extratemporal lobe epilepsy groups) $(52.9 \%)\left(\chi^{2}=7.0, P=0.008\right)$. When the individuals with lesions were subdivided into those with mesial temporal lobe epilepsy (50.4\%) and non-mesial temporal sclerosis lesions (temporal and extratemporal lobe epilepsy groups) (56\%) and the groups compared individually with the non-lesional group, both had a lower prevalence of psychiatric disorders than those with nonlesional focal epilepsy (temporal and extratemporal lobe epilepsy groups) $(69.2 \%)\left(\chi^{2}=7.7, P=0.021\right)$.

There was no statistical association between the prevalence of a psychiatric diagnosis with the laterality of the seizure focus (right 56.3\%, left 61\%, bilateral 54.5\%) $\left(\chi^{2}=0.8, P=0.7\right)$. There were no significant differences in the prevalence of any psychiatric disorder between patients with temporal lobe epilepsy (57\%) $v$. extratemporal lobe epilepsy $(62.1 \%)\left(\chi^{2}=0.58, P=0.49\right)$. People with non-lesional epilepsy (both temporal and extratemporal lobe epilepsy groups) had a significantly higher prevalence of psychiatric disorders $(69.2 \%)$ than those with lesional epilepsy (mesial temporal sclerosis lobe epilepsy, lesional temporal lobe epilepsy, and lesional extratemporal lobe epilepsy groups; 52.9\%) $\left(\chi^{2}=7.07, P=0.008\right)$.

Logistic regression analysis identified lesional $v$. non-lesional epilepsy as the only independent variable to be related statistically to the diagnosis of a psychiatric illness $(P=0.006)$ (Table 2). A contrast comparing lesional focal epilepsy with non-lesional focal epilepsy was significant $(P=0.002)$. The odds of any psychiatric diagnosis for those with non-lesional focal epilepsy were 2.4 times the odds for individuals with lesional focal epilepsy. There were no statistical differences according to whether the lesion was mesial temporal sclerosis or another lesion, or according to laterality of the seizure focus.

\section{Prevalence of depressive symptoms}

The results for past or current depressive symptoms were similar to the results for any psychiatric diagnosis. There were no significant differences in the prevalence of depression between individuals with temporal lobe epilepsy (31.2\%) and extratemporal

\begin{tabular}{|c|c|c|c|c|}
\hline \multirow[b]{2}{*}{ Independent variables } & \multicolumn{2}{|c|}{ Any psychiatric diagnosis } & \multicolumn{2}{|c|}{ Diagnosis of depression } \\
\hline & $P$ & OR $(95 \% \mathrm{Cl})$ & $P$ & OR $(95 \% \mathrm{Cl})$ \\
\hline Focus (TLE/ETLE) & 0.8 & $0.90(0.46-1.77)$ & 0.5 & $0.78(0.40-1.54)$ \\
\hline Lesional $v$. non-lesional & 0.006 & & 0.04 & \\
\hline MTS and other lesions $v$. non-lesional & 0.002 & $2.38(1.37-4.13)$ & 0.01 & $1.96(1.16-3.31)$ \\
\hline MTS $v$. other lesions & 0.5 & $1.26(0.69-2.30)$ & 0.6 & $1.17(0.60-2.27)$ \\
\hline Laterality & 0.6 & & $>0.9$ & \\
\hline Right- $v$. left-sided foci & 0.4 & $1.23(0.76-2.00)$ & 0.9 & $1.05(0.63-1.74)$ \\
\hline Right and left $v$. bilateral foci & 0.7 & $0.82(0.33-2.07)$ & 0.8 & $0.91(0.35-2.38)$ \\
\hline
\end{tabular}


lobe epilepsy $(37.9 \%)\left(\chi^{2}=1.06, P=0.3\right)$, nor between those with right-sided $(32.6 \%)$, left-sided $(33.1 \%)$ or bilateral $(31.8 \%)$ laterality $\left(\chi^{2}=0.2, P=0.99\right)$. Groups with non-lesional focal epilepsy exhibited a higher prevalence of depression (41.6\%) compared with those with a lesion on MRI (mesial temporal lobe epilepsy, temporal and extratemporal lobe epilepsy groups) (28.5\%) $\left(\chi^{2}=5.17, P=0.03\right)$. There was no significant difference in the rate of depression between individuals with mesial temporal sclerosis (26.4\%) and those with other types of focal lesions in the temporal lobe $(27.6 \%)\left(\chi^{2}=0.026, P=0.86\right)$.

Logistic regression analysis confirmed a diagnosis of depression was best predicted by non-lesional epilepsy $(P=0.04)$ when site of seizure onset, lesional epilepsy and laterality were included in the model (Table 2). This was confirmed by examining lesional epilepsy compared with non-lesional epilepsy $(P=0.01)$. The odds of a diagnosis of depression for those with non-lesional focal epilepsy were nearly double the odds for those with lesional focal epilepsy ( $\mathrm{OR}=1.96,95 \%$ CI 1.16-3.31) A diagnosis of depression was not predicted by whether the focus was temporal or extratemporal $(P=0.5)$. Furthermore, there was no statistical difference between rates of depression in those with mesial temporal lobe epilepsy compared with other lesions $(P=0.6)$. A diagnosis of depression was not predicted by site of seizure onset or the laterality of focus.

To determine if depression was the most important factor in the association of non-lesional epilepsy with 'any psychiatric diagnosis', the analyses were repeated with all individuals with depression excluded. This analysis did not show any association between the independent variables and no longer showed significant differences for non-lesional epilepsy. This indicates that the major contributor to the pattern of results from the first set of analyses (for 'any psychiatric diagnosis') was a history of depressive symptoms.

\section{Prevalence of psychosis}

There were no significant relationships between any of the epilepsy variables and a history of past or current psychotic symptoms. The analyses comparing temporal lobe epilepsy with extratemporal lobe epilepsy, lesional compared with non-lesional focal epilepsy, mesial temporal sclerosis compared with other lesional and nonlesional focal epilepsy and the laterality of the seizure focus revealed no associations on univariate $\chi^{2}$ analysis. Logistic regression similarly showed no significant association for any of the variables (Table 3 ).

\section{Discussion}

The strength of this study lies in its combination of rigorous, modern epilepsy syndrome classification and clinically relevant psychiatric diagnoses in a large consecutive cohort of individuals with medically refractory focal epilepsy. There were three major findings of this study related to the prevalence of psychiatric diagnoses across focal epilepsy subtypes, the effect of whether it was lesional or non-lesional epilepsy, and the effect of whether it was right-sided, left-sided or bilateral.

First, the prevalence of psychiatric disorder does not differ between people with temporal lobe epilepsy and extratemporal lobe epilepsy. Furthermore, individuals with medial temporal lobe epilepsy did not exhibit higher rates of psychiatric diagnoses when compared with those with other temporal lobe epilepsy (i.e. non-lesional temporal lobe epilepsy) or extratemporal lobe epilepsy. This finding is in keeping with a number of studies over the years which have failed to confirm the commonly held view that there is a specific association between temporal lobe epilepsy and psychopathology ${ }^{15-17}$ but in contrast to commonly accepted clinical practise. Second, people with no identified lesion on MRI were more likely to have a current or lifetime history of depression. This finding was independent of whether the seizure focus was temporal or extratemporal. Third, psychiatric diagnoses were not related to whether the site of the epilepsy focus was right-sided, left-sided or bilateral.

Studies that have identified higher prevalence rates of psychopathology in temporal lobe epilepsy in general did not employ MRI and VEM for epilepsy diagnosis and had smaller numbers of participants than our study. ${ }^{25-27}$

We were not able to confirm the findings of Quiske et al who found significantly higher depression scores on the Beck Depression Inventory $(\mathrm{BDI})^{28}$ in people with mesial temporal sclerosis compared with those with lesions in other temporal neocortical regions. ${ }^{29}$ Their paper excluded individuals with extratemporal lobe epilepsy and non-lesional focal epilepsy, comparing people with temporal lobe epilepsy and mesial temporal sclerosis with those with temporal lobe epilepsy and neocortical lesions. However, when we performed a similar analysis on our cohort, mesial temporal lobe epilepsy $v$. lesional temporal lobe epilepsy, we found no statistical differences in rates of psychiatric disorders. There are a number of differences in the studies which may account for this discrepancy. Our sample size was larger (i.e. 179 people, of whom 121 had mesial temporal lobe epilepsy and 58 had lesional temporal lobe epilepsy) than that of the previous study (i.e. 60 people, of whom 43 had mesial temporal sclerosis and 16 had neocortical temporal lesions), making the chance of a Type II error less likely. As our data were undertaken prospectively for clinical purposes we do not have BDI scores. Although clinical assessment is likely to be more accurate in this population than a BDI, they are not equivalent assessments. Also, owing to data collection limitations we have both mesial and neocortical 'alien tissue' lesions within our lesional temporal lobe epilepsy group. This may obscure a true difference dependent on site within the temporal lobe rather than the nature of the pathology. In addition, the mesial temporal sclerosis group in the Quiske study had a significantly

\begin{tabular}{|c|c|c|c|c|}
\hline \multirow[b]{2}{*}{ Independent variables } & \multicolumn{2}{|c|}{ Diagnosis of psychosis } & \multicolumn{2}{|c|}{ Diagnosis of personality disorder } \\
\hline & $P$ & OR $(95 \% \mathrm{Cl})$ & $P$ & OR $(95 \% \mathrm{Cl})$ \\
\hline Focus (TLE/ETLE) & 0.6 & $1.5(0.37-5.96)$ & 0.5 & $1.38(0.56-3.38)$ \\
\hline Lesional v. non-lesional & 0.9 & & 0.5 & \\
\hline MTS and other lesions $v$. non-lesional & 0.7 & $0.8(0.28-2.29)$ & 0.3 & $1.38(0.74-2.59)$ \\
\hline MTS $v$. other lesions & 0.9 & $1.1(0.35-3.38)$ & 0.4 & $0.73(0.33-1.62)$ \\
\hline Laterality & 0.9 & & 0.6 & \\
\hline Right- $v$. left-sided foci & 0.7 & $0.84(0.34-2.09)$ & 0.5 & $1.26(0.68-2.33)$ \\
\hline Right and left $v$. bilateral foci & 0.7 & $0.7(0.09-5.53)$ & 0.5 & $1.52(0.52-4.42)$ \\
\hline
\end{tabular}


earlier seizure onset and longer duration of illness than their neocortical lesional group. If mesial temporal sclerosis and other focal epilepsies are progressive conditions, ${ }^{30}$ psychiatric progression may explain the increased incidence of depression in this particular mesial temporal sclerosis group. Unfortunately we were unable to test for such an association in our cohort, because of lack of availability of reliable data about epilepsy onset.

The association between depression and non-lesional epilepsy, regardless of whether the individual had temporal or extratemporal lobe epilepsy, is a novel finding that has not previously been reported. Psychosis rather than depression is usually associated with the presence or absence of lesions. It has been generally accepted from neuropathological studies that foreign tissue lesions may have higher rates of psychosis than mesial temporal sclerosis ${ }^{31}$ and that lesions of perinatal origin may be especially relevant. ${ }^{32}$ Although there is no study looking at the importance of lesional $v$. non-lesional epilepsy in depression, there are several compelling reasons to do so. First, such an approach classifies the participants according to the nature of the underlying epileptogenic pathology. Second, Roberts et al did not investigate a relationship between groups with and without lesions and depression. ${ }^{32}$ The failure of previous studies to investigate whether there is an association between having either lesional or non-lesional epilepsy and psychopathology is understandable given that the majority of studies have tried to understand psychopathology by investigating the site of the seizure focus. The results of this study suggest that the nature of the underlying pathological abnormality may be of greater significance than its localisation. We found no association between the focal site and the prevalence of psychiatric illness.

Although the current study identifies a relationship between the prevalence of depression and non-lesional focal epilepsy, the neurobiological basis of this finding remains speculative. The non-lesional group may represent individuals with subtle diffuse changes or lesions not detectable on MRI (owing to resolution limitations). Supportive of the proposition that these individuals have more diffuse pathology is the more widespread PET hypometabolism seen in those with temporal lobe epilepsy without an MRI lesion compared with those with mesial temporal sclerosis. ${ }^{33}$ Furthermore, the literature suggests that more extensive functional impairment is associated with the presence of psychiatric disorders. People with epilepsy and with depression $^{34,35}$ and psychosis, ${ }^{36}$ compared with those without psychiatric comorbidity, have more extensive functional imaging changes in cerebral regions such as the frontal lobes. While focal lesions may result in the complete interruption of neuronal connections at the site of the lesion, diffuse pathology may result in more widespread disruption of frontolimbic pathways. ${ }^{32}$ If people with non-lesional epilepsy have more extensive functional impairment than those with lesional focal epilepsy, this may account for the increased rates of depression. If confirmed, this finding would have potential implications for the management and rehabilitation of these people.

We did not identify any significant associations between the epilepsy subgroups and the prevalence of psychosis, nor did we replicate the previously reported findings of increased rates of psychosis in those with lesions despite investigating similar numbers of participants. ${ }^{32}$ It must be acknowledged that small numbers in all studies make conclusions in this context difficult to establish.

\section{Strengths}

Strengths of the current study relate to the use of VEM and MRI to ascertain focal epilepsy type, the large sample size across focal epilepsy subtypes, the inclusion of individuals with extratemporal and non-lesional focal epilepsy. The use of clinical neuropsychiatric assessments to identify psychiatric morbidity may be considered a limitation of the study compared with studies which use standardised diagnostic tools. However, clinical neuropsychiatric assessments in our service are more thorough and more likely to detect atypical symptoms that a standardised rating scale. Full neuropsychiatric formulation is generally accepted to be the gold standard for psychiatric diagnosis. Given that the aim of the study was to identify clinically relevant psychiatric diagnoses in a large cohort of individuals with focal epilepsy, the availability of clinical assessments by a single experienced neuropsychiatrist over an 11year period is unique in the literature, with respect to the number of individuals assessed, the depth of a clinical assessment and the continuity of assessment. Clinical interview by an experienced clinician may be a more valid diagnostic tool in this population given the atypical nature of psychiatric presentations and the recognised deficiencies of DSM diagnostic criteria. ${ }^{37}$

\section{Limitations}

There are several limitations in this study which are inherent in retrospective studies of clinical populations. First, despite studying a large cohort, the numbers in some individual subgroups, especially those with psychosis, are relatively small. This limitation is not unique to our study. Most studies investigating the psychoses of epilepsy have similar numbers. ${ }^{6,18,32}$

Second, the retrospective design did not allow the standardised assessment of variables previously associated with psychiatric comorbidity such as the effects of antiepileptic medication, duration of illness, presence of febrile convulsions or frequency and clustering of seizures.

Third, there are inevitable elements of selection bias in our series. All individuals were referred to a tertiary treating centre with medically refractory focal epilepsy and can therefore only be seen as representative of those with chronic illness. In addition, although all individual who were admitted were referred, not all were seen by the neuropsychiatrist. While our review of the medical files suggests that neuropsychiatrist leave was responsible for non-assessments, the possibility remains that individuals perceived by referrers to have psychiatric illness may have been preferentially referred. In addition, participants admitted with temporal lobe epilepsy for surgery may also have been preferentially referred. In order to address this issue we undertook a sensitivity (or intention to treat) style analysis including all individuals in the cohort and presuming those not seen had no psychiatric diagnosis. Those with temporal lobe epilepsy and extratemporal lobe epilepsy were then compared again for a psychiatric diagnosis and again there was no significant difference found between rates $\left(\chi^{2}=0.582, P=0.446\right)$. We conclude therefore that this mild assessment imbalance has not artificially hidden a real difference between these groups in rates of psychiatric illness. Importantly, the neuropsychiatric assessment was undertaken prior to the epilepsy clinical review meeting in which the participants' epilepsy syndromes were classified and thus was formulated without knowledge of the formal focal epilepsy diagnosis.

The use of VEM and MRI to identify focal epilepsy type in a large number of individuals has allowed us to re-evaluate many of the previously reported associations of psychiatric disorders and focal epilepsy. The study has failed to confirm associations between depression and male gender, left-sided laterality or mesial temporal sclerosis. We did not identify any associations between the prevalence of psychosis or personality disorders and focal epilepsy subtypes.

The study's novel finding was of increased rates of depression in non-lesional focal epilepsy, independent of the lobe of seizure 
focus. We hypothesise that this association is related to the presence of a more diffuse underlying epileptogenic pathogenic process in these individuals. Further clarification of these issues will have significant clinical implications for people with focal epilepsy and may potentially influence our understanding of the underlying neurobiology of major psychiatric illnesses such as psychosis and depression.

Sophia J. Adams, MBBS, MA(Bioethic), FRANZCP, Melbourne Neuropsychiatry Centre, University of Melbourne and Royal Melbourne Hospital, Melbourne; Terence J. O'Brien, MBBS, MD, FRACP, Departments of Medicine and Surgery, University of Melbourne and Department of Neurology, Royal Melbourne Hospital; John Lloyd, MBBS, FRANZCP, Neuropsychiatry Unit, Royal Melbourne Hospital; Christine J. Kilpatrick, MBBS, MD, FRACP, Royal Melbourne Hospital - Melbourne Health; Michael R. Salzberg, MBBS, FRANZCP, St Vincent's Hospital, Melbourne; Dennis Velakoulis, MBBS, FRANZCP, Melbourne Neuropsychiatry Centre, University of Melbourne and Royal Melbourne Hospital, Melbourne, Australia

Correspondence: Sophia J. Adams, Melbourne Neuropsychiatry Centre, The University of Melbourne and Royal Melbourne Hospital, Level 2, John Cade Building, Melbourne, Australia. Email: Sophia.Adams@mh.org.au

First received 22 Oct 2007, final revision 30 Jan 2008, accepted 27 Feb 2008

\section{Acknowledgements}

The statistical analysis was conducted by S.A., with support from Sue Finch, PhD, Department of Mathematics and Statistics, University of Melbourne.

\section{References}

1 Gibbs FA, Gibbs EL, Furster B. Psychomotor epilepsy. Arch Neurol Psychiatry 1948; 60: 331-9.

2 Slater E, Beard AW. The schizophrenia-like psychoses of epilepsy, V: Discussion and conclusions. 1963. J Neuropsychiatry Clin Neurosci 1995; 7 372-8.

3 Flor-Henry P. Psychosis and temporal lobe epilepsy. A controlled investigation. Epilepsia 1969; 10: 363-95.

4 Gaitatzis A, Trimble MR, Sander JW. The psychiatric comorbidity of epilepsy Acta Neurol Scand 2004; 110: 207-20.

5 Sachdev P. Schizophrenia-like psychosis and epilepsy: the status of the association. Am J Psychiatry 1998; 155: 325-36.

6 Torta R, Keller R. Behavioral, psychotic, and anxiety disorders in epilepsy: etiology, clinical features, and therapeutic implications. Epilepsia 1999; 40 (suppl 10): s2-20.

7 Lishman WA. Organic Psychiatry. Blackwell Science, 1998.

8 Lambert MV, Schmitz B, H. Ring, Trimble M. Neuropsychiatry. Lippincott Williams \& Wilkins, 2003

9 Victoroff JI. DSM-III-R Psychiatric diagnoses in candidates for epilepsy surgery: lifetime prevalence. Neuropsychiatry Neuropsychology Behav Neurol 1994; 7: 87-97.

10 Victoroff ال I, Benson F, Grafton ST, Engel J, Jr. Mazziotta JC. Depression in complex partial seizures. Electroencephalography and cerebral metabolic correlates. Arch Neurol 1994; 51: 155-63.

11 Swinkels WAM, Kuyk J, van Dyck R, Spinhoven P. Psychiatric comorbidity in epilepsy. Epilepsy Behav 2005; 7: 37-50.

12 Edeh J, Toone B. Relationship between interictal psychopathology and the type of epilepsy. Results of a survey in general practice. Br J Psychiatry 1987 151: 95-101.

13 Hesdorffer DC, Hauser WA, Annegers JF, Cascino G. Major depression is a risk factor for seizures in older adults. Ann Neurol 2000; 47: 246-9.
14 Qin P, Xu H, Laursen TM, Vestergaard M, Mortensen PB. Risk for schizophrenia and schizophrenia-like psychosis among patients with epilepsy: population based cohort study. BMJ 2005; 331: 23.

15 Stevens JR. Psychiatric aspects of epilepsy. J Clin Psychiatry 1988; 49 (suppl): 49-57.

16 Manchanda R, Schaefer B, McLachlan RS, Blume WT. Interictal psychiatric morbidity and focus of epilepsy in treatment-refactory patients admitted to an epilepsy unit. Am J Psychiatry 1992; 149: 1096-8.

17 Swinkels WA, Kuyk J, de Graaf EH, van Dyck R, Spinhoven P. Prevalence of Psychopathology in Dutch Epilepsy Inpatients: A Comparative Study. Epilepsy Behav 2001; 2: 441-7.

18 Parnas J, Korsgaard S. Epilepsy and psychosis. Acta Psychiatr Scand 1982 66: 89-99.

19 Stevens JR. Psychiatric implications of psychomotor epilepsy. Arch Gen Psychiatry 1966; 14: 461-71.

20 Small JG, Small IF, Hayden MP. Further psychiatric investigations of patients with temporal and nontemporal lobe epilepsy. Am J Psychiatry 1966; 123 303-10.

21 Standage KF, Fenton GW. Psychiatric symptom profiles of patients with epilepsy: a controlled investigation. Psychol Med 1975; 5: 152-60.

22 Lowe AJ, David E, Kilpatrick CJ, Matkovic Z, Cook MJ, Kaye A, O’Brien TJ. Epilepsy surgery for pathologically proven hippocampal sclerosis provides long-term seizure control and improved quality of life. Epilepsia 2004; 45: 237-42.

23 International League Against Epilepsy. Proposal for classification of the epilepsies and epileptic syndromes. Epilepsia 1985; 26: 268-78.

24 American Psychiatric Association. Diagnostic and Statistical Manual of Mental Disorders (4th edn) (DSM-IV). APA, 1994.

25 Gureje 0 . Interictal psychopathology in epilepsy. Prevalence and pattern in a Nigerian clinic. Br J Psychiatry 1991; 158: 700-5.

26 Gudmundsson G. Epilepsy in Iceland. A clinical and epidemiological investigation. Acta Neurol Scand 1966; 43 (suppl 25): 1-124.

27 Rodin EA, Katz M, Lennox K. Differences between patients with temporal lobe seizures and those with other forms of epileptic attacks. Epilepsia 1976; 17: 313-20.

28 Beck AT, Ward $\mathrm{CH}$, Mendelson M, Mock J, Erbaugh J. An inventory for measuring depression. Arch Gen Psychiatry 1961; 4: 561-71.

29 Quiske A, Helmstaedter C, Lux S, Elger CE. Depression in patients with temporal lobe epilepsy is related to mesial temporal sclerosis. Epilepsy Res 2000; 39: 121-5.

30 Sutula TP, Hagen J, Pitkanen A. Do epileptic seizures damage the brain? Curr Opin Neurol 2003; 16: 189-95.

31 Taylor DC. Mental state and temporal lobe epilepsy. A correlative account of 100 patients treated surgically. Epilepsia 1972; 13: 727-65.

32 Roberts GW, Done DJ, Bruton C, Crow TJ. A 'mock up' of schizophrenia: temporal lobe epilepsy and schizophrenia-like psychosis. Biol Psychiatry 1990; 28: 127-43.

33 Carne RP, O'Brien TJ, Kilpatrick CJ, MacGregor LR, Hicks RJ, Murphy MA Bowden SC, Kay AH, Cook MJ. MRI-negative PET-positive temporal lobe epilepsy: a distinct surgically remediable syndrome. Brain 2004; 127 2276-85.

34 Salzberg $M$, Taher T, Davie M, Carne R, Hicks RJ, Cook M, Murphy M, Vinton A, O'Brien TJ. Depression in temporal lobe epilepsy surgery patients: an FDGPET study. Epilepsia 2006; 47: 2125-30.

35 Bromfield EB, Altshuler L, Leiderman DB, Balish M, Ketter TA, Devinsky O, Post RM, Theodore WH. Cerebral metabolism and depression in patients with complex partial seizures. Arch Neurol 1992; 49: 617-23.

36 Gallhofer B, Trimble MR, Frackowiak R, Gibbs J, Jones T. A study of cerebral blood flow and metabolism in epileptic psychosis using positron emission tomography and oxygen. J Neurol Neurosurg Psychiatry 1985; 48: 201-6.

37 Blumer D, Montouris G, Hermann B. Psychiatric morbidity in seizure patients on a neurodiagnostic monitoring unit. J Neuropsychiatry Clin Neurosci 1995; 7: $445-56$. 